\title{
An intelligent method using neural networks for Depth detection by standard thermal contrast in active thermography
}

\author{
by H.Halloua*, A.Elhassnaoui ${ }^{* *}$, A.Saifi ${ }^{\star}$, A.Obbadi ${ }^{\star}, Y . E r r a m i{ }^{*}$, S. Sahnoun*
}

*Laboratory of Electronics, Instrumentation and Energetic, Faculty of Sciences, B.P 20. 24000 El Jadida,

Morocco.ssahnoun@gmail.com

${ }^{* *}$ Industrial Engineering Laboratory, Faculty of Science and Technology, BP: 523 Beni Mellal, Morocco

\begin{abstract}
Today the infrared thermography is among the nondestructive testing methods (NDT) most used for detection and characterization of internal defects in materials. It has become a reference method in industrial installations control. As the interpretation of thermal images provided by the infrared cameras is often difficult; therefore, it is necessary; to seek new methods fast and reliable for intelligent nondestructive evaluation. In our work we propose a fast method using artificial neural networks for internal defects depth evaluation from the thermal contrast. Experimental results have confirmed the method efficiency in predicting the defects depths.
\end{abstract}

Keywords: Infrared radiation, artificial neural network, defect depth, pulsed thermography, finite element Method.

\section{Introduction}

The use of artificial intelligence methods, such as neural networks in the characterization of subsurface defects is a current topic of several research works. In [1] Henrique Fernandes used a neural network to estimate the orientation of the fibers in a carbon fiber reinforced polymer (CFRP). In [2] Nazmul Huda has used a perceptron neural network for an intelligent method to predict invisible thermal defects in electrical equipment. In [3] and [4] S. Dudzik has used an artificial neural network algorithm in defect detection and estimation of their depths with thermal image processing. He made principal components analysis (PCA) to reduce the number of neural networks inputs. He used fifteen principal components for each recorded sequence. For processing of thermal data used in the training stage, this method required a lot of operation and a lot of computing time.

In this work we exploit the temperature value at a single moment to characterize the depth of the subcutaneous defects. This new method represents a considerable gain in memory space and data processing time. The experimental results confirmed the relevance and performance of the proposed method.

\section{Pulsed Infrared thermography}

Pulsed thermography is the most popular methods of active Thermal Control, it consists in excite the inspected sample by a thermal pulse and analyze its thermal response (figure 1). The inspected sample response is recorded with an infrared camera in digital form for careful analysis to characterize the existing defects $[5,6,7,8]$. There are several methods in the literature who studied the thermal responses samples for determining the geometric characteristics of the defects such as depth and size.

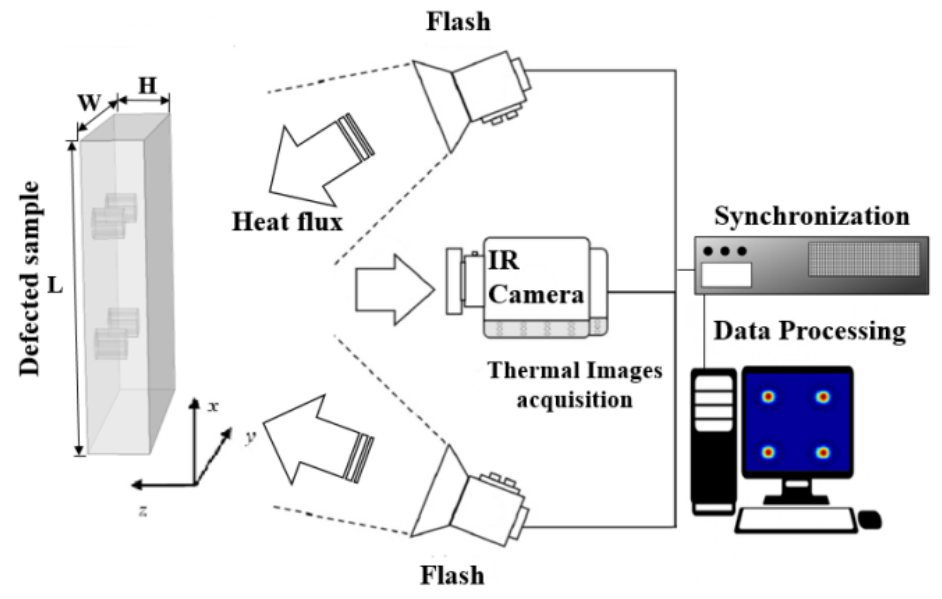

Fig. 1. Pulsed thermography principle 


\subsection{1/qirt.2016.110}

Among these methods, there are recent methods in the nondestructive testing field, there are intelligent methods using neural networks

\section{Artificial neural networks}

An Artificial neural network is a data processing technique inspired by the functioning of biological neurons. It is part of the toolbox for researchers who want to make predictions, mathematical models, recognitions geometric shapes of objects etc.

The neural network topology is defined by its architecture and its connections nature, it can be described by the layers number and number of neurons in each layer (figure 2). To have a good result in a short time. it must optimize the layer number and reduce neurons number.

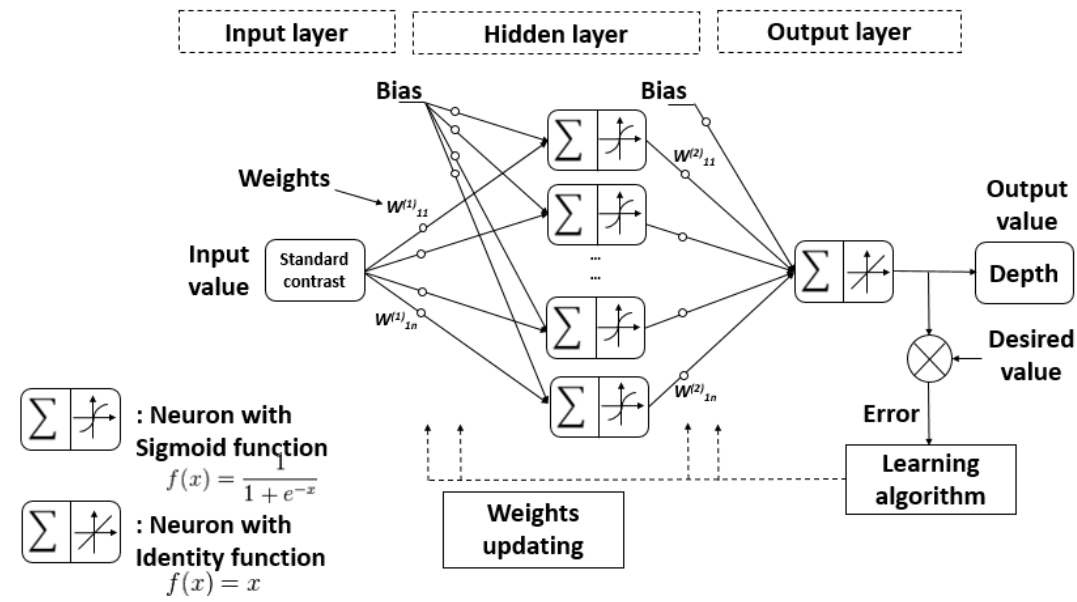

Fig. 2. Neural network with three layers : depth estimator from standard contrast value at instant $t_{a} C\left(t_{a}\right)$.

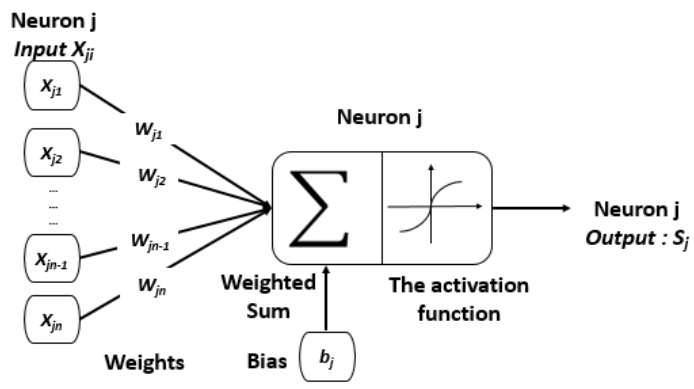

Fig. 3. Mathematical representation of formal neuron $j$ with $n$ inputs $\left(x_{j i}\right)$ and one output $\left(S_{j}\right)$.

Neural networks are composed of interconnected elemental data processing units called formal neurons (figure 3 ); they are able to learn from their input information $\left(\mathrm{x}_{\mathrm{ji}}\right)$, and perform logical, arithmetic and symbolic complex operations to get in its output $\left(\mathrm{S}_{\mathrm{j}}\right)$ the desired result.

\subsection{The formal neuron}

The formal neuron can be represented by a cell with multiple inputs and one output, and it can be modelled by two operators:

- $\quad$ a summing operator who develops a " postsynaptic potential " $p_{j}$ of neuron number $j$ equal to the weighted sum

of the cell inputs plus a constant term called bias that may be considered as the weight of $x_{0}$ for entry equal to 1 :

$$
p_{j}=b_{j}+\sum_{\mathrm{i}=1}^{\mathrm{n}} w_{j i} x_{j i}
$$

Where, $x_{\mathrm{ji}}$ describe the neuron number $\mathrm{j}$ inputs and $\mathrm{w}_{\mathrm{ii}}$ is the connections weights.

- a decision operator $f\left(p_{j}\right)$ calculates the output state $\left(s_{j}\right)$ of the neuron according to its potential $p_{j}$; this operator is called "the activation function":

$$
s_{j}=\mathrm{f}\left(p_{j}\right)
$$


The neuron state is obtained by calculating the post-synaptic potential with the decision operator. The calculation is called neuron update [9].

\subsection{Neural Networks Learning}

The network learning is made by synaptic weights $\left(w_{\mathrm{ji}}\right)$ modifications until the desired result is obtained. There are two major types of learning: The supervised learning in which the network is forced to converge towards a specific end state, by comparing outputs with the expected targets (learning with a teacher). It is a suitable learning for function approximations.

The second is the unsupervised learning (or self-organizational) where the network is left free to converge to any final state. In this case, the network will change as the statistical regularities and correlations that it discovered in the learning inputs (Redundancy Provides knowledge). This last type of learning is suitable for tasks and associative groupings.

On the basis of learning rules, the network weights modifications are automated by the use of trainings algorithms. The backpropagation algorithm of the gradient error developed by D.Rumelhart [10] is currently the most used in neural networks supervised learning. It minimizes an error function "E" expressed from the squared errors, for an input-output pair $\left(\mathrm{d}_{\mathrm{k}, \mathrm{Sk}}\right)$ of the neuron with the index $\mathrm{k}$ Eq. (3).

$$
\mathrm{E}=\frac{1}{2} \sum_{\mathrm{k}=0}^{\mathrm{n}}\left(d_{k}-s_{k}\right)^{2}
$$

The algorithm propagates the errors from layer to layer according to the importance of the elements that produced errors. This process is repeated for several iterations until converging to a very low error value $\mathrm{E}$.

It is dangerous to continue incessantly the learning phase without control; the training phase of a neural network is made, when the obtained synaptic weights provide the desired data without error. At this stage, in fact, the neural network is not wrong in his predictions, but there is a huge risk that these predictions are accurate for only the data on which the learning was based. This is called overfitting. We prevent the overfitting by dividing the data into two: training data and validation data. The training data will be used in the algorithm which provides the best values of synaptic weights. The validation data do not intervene in weights determining; They stop learning in the iteration preceding the overlearning.

In practice, we divide the learning data into three data sets: the training phase part, the validation part and a last one for the testing phase. The learning phase is often stopped when the calculated error becomes small. After the neural network is well trained (after learning), it is necessary to test it on a different database than those used in learning. This test allows both to assess the neural network performance and detect the type of data that cause problems [11].

\section{Principle of the method}

\subsection{The model used}

To apply the neural network in estimating defects depths; we consider an aluminum sample with dimensions 350 $\mathrm{mm} \times 200 \mathrm{~mm} \times 15 \mathrm{~mm}$ (figures 4 and 5), this sample contains triangular defects located at various depths ranging from 2 to $7 \mathrm{~mm}[12]$. The duration of the heat pulse is few microseconds.

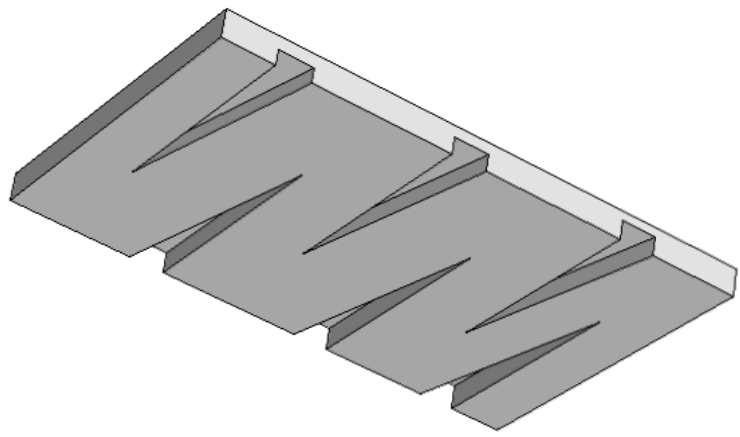

Fig4. $3 d$ view of controlled sample

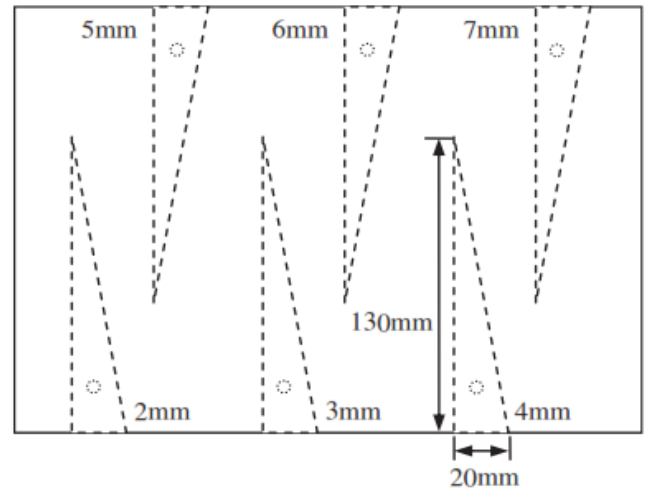

Fig5. $2 d$ view of controlled sample

\subsection{The standard thermal contrast:}

The use of the standard thermal contrast to reveal the presence of defects is very wise for several reasons. The standard thermal contrast $\mathrm{C}(\mathrm{t}) \mathrm{Eq}$. (4) is defined as the ratio between the defective area temperatures differences and those of the non-defective area, these temperature differences are calculated between the instants $t$ and to [13]:

$$
\mathrm{C}(\mathrm{t})=\frac{T_{d}(\mathrm{t})-T_{d}\left(t_{0}\right)}{T_{s}(\mathrm{t})-T_{s}\left(t_{0}\right)}
$$




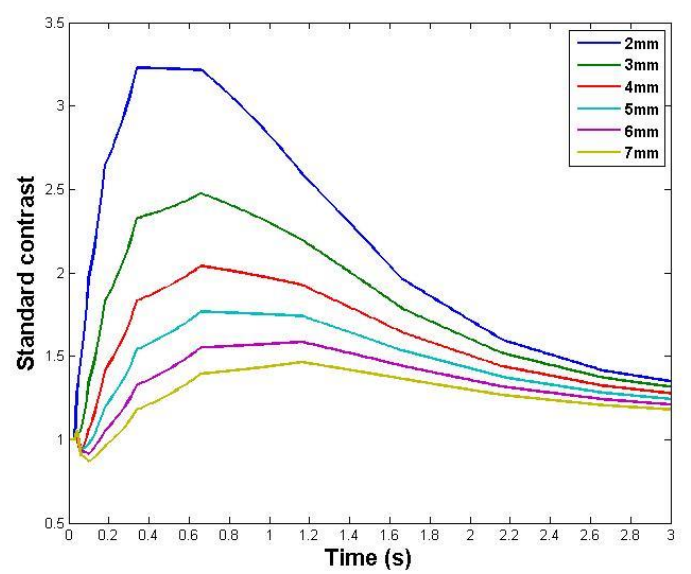

Fig. 6. Contrast variation with time

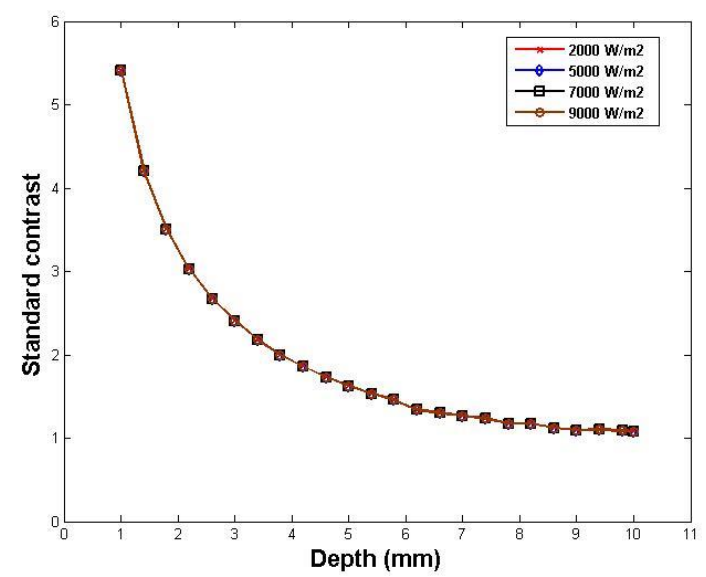

Fig. 7. Contrast variation with depth at $t_{a}=0.5 \mathrm{~s}$

The figure 6 shows the standard thermal contrast variation with time for ranging depths from $2 \mathrm{~mm}$ to $7 \mathrm{~mm}$. We can notice a correspondence between the depth of the defects and the thermal contrast at all measurement times. We notice that the standard thermal contrast reached important values in the time interval [0.4s, $1.4 \mathrm{~s}]$. In our work we will take as measurement instant a moment that belongs to this range. We took the instant $t_{a}=0.5 \mathrm{~s}$.

We reported in figure7 the standard thermal contrast variation with depth at the instant $t_{a}=0.5 \mathrm{~s}$ for different heat flux densities. We notice that the curves are superposed; so the standard thermal contrast is independent of the applied heat power, this represents a considerable advantage of using it in neuronal method. We also remark that for depths superior to $8 \mathrm{~mm}$, the contrast is almost the same and tends to 1, which shows the limit of the pulsed thermography application for deep defects.

According to the figure 7 curve shape, we will use non-linear regression between the defect depth and the thermal contrast at time ta. For that, we will use neural networks to deal those problems.

\subsection{The chosen neural network}

In this work, a feed forward artificial neural network is used to determine the defect depth; we used a supervised learning because it is the most suitable for functions approximations. By the finite element method at the instant $t_{a}=0.5 \mathrm{~s}$, we calculated the standard thermal contrast (Eq. 1) for 560 depths, ranging between $1 \mathrm{~mm}$ and $10 \mathrm{~mm}$, they are used as the network training inputs values. The outputs are the corresponding depths contrasts chosen on entrance. We divided the 560 learning couples (contrast-depth) according to the percentage $70 \%$ for network training, $15 \%$ for validation and $15 \%$ for testing the network performance.

The neural network structure choice represents a very important step; it consists on setting the number of network layers, the number of neurons, the activation function and the learning algorithm to use.

To set the used number of layers and the activation function, we relied on the work of Cybenko and Funahashi [14] which showed that a single hidden layer using sigmoid activation functions is sufficient to approximate any non-linear function. It must be noted that the neurons of the input and the output layers are not really computing neurons, but they have the utility to normalize the input signals distribution and the output signals compilation, the identity function is used on the neurons of this latter as activation function.

We used an incremental approach where we start the learning with a minimal number of neurons (equal to one) in the hidden layer, then adding each time a new neuron and comparing the error of the new network with the previous one. The process ends when adding a new neuron will not improve the precision. The neuronal performance is calculated by the mean square error between the real output values $s_{i}$ and the desired values $d_{i}(E q .5)$.

$$
\mathrm{mse}=\frac{1}{\mathrm{n}} \sum_{\mathrm{i}=0}^{\mathrm{n}}\left(d_{i}-s_{i}\right)^{2}
$$




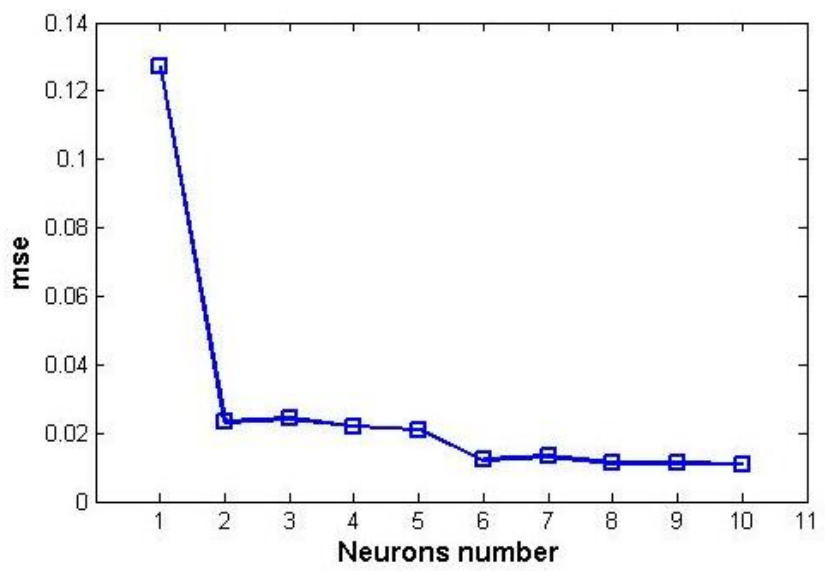

Fig. 8. Effect of hidden layer neurons number of the on network performance

This method allows the use of networks with reduced neurons number in a small interval of learning time. In our case the use of 10 neurons gave the best results (figure 8).

To select the learning algorithm that we will adopt, we compared the results of 14 types of backpropagation algorithm among the most frequently used (table 1). We found that the Levenberg-Marquardt algorithm gave the best result. It gave a low mse gap with a minimum of iterations.

Table 1. Performance of the different backpropagation algorithms tested in the learning process.

\begin{tabular}{|l|c|c|c|}
\hline \multicolumn{1}{|c|}{ Backpropagation algorithm } & Acronym & mse & iterations \\
\hline LevenbergMarquardt & TRAINLM & 0.010908 & 24 \\
\hline BFGS Quasi-Newton & TRAINBFG & 0.16383 & 23 \\
\hline ResilientBackpropagation & TRAINRP & 0.04997 & 236 \\
\hline ScaledConjugate Gradient & TRAINSCG & 0.03201 & 57 \\
\hline Conjugate Gradient with Powell/Beale Restarts & TRAINCGB & 0.02483 & 82 \\
\hline Fletcher-Reeves Conjugate Gradient & TRAINCGF & 0.02644 & 100 \\
\hline Polak-RibiéreConjugateGradient & TRAINCGP & 0.50123 & 34 \\
\hline One StepSecant & TRAINOSS & 0.53158 & 22 \\
\hline Bayesianregulation & TRAINBR & 5.8053 & 1000 \\
\hline Gradient Descent & TRAINGD & 0.04818 & 1000 \\
\hline Gradient Descent with Adaptative learning rate & TRAINGDA & 0.08571 & 164 \\
\hline Gradient Descent with Momentum & TRAINGDM & 4.1565 & 115 \\
\hline Gradient Descent with Momentum and Adaptative learning rate & TRAINGDX & 1.2099 & 8 \\
\hline RandomWeight/BiasRule & TRAINR & 9.6076 & 1000 \\
\hline
\end{tabular}

\subsection{The simulation results:}

We reported in figure 9 the mean squared error (mse) variations obtained by the Levenberg Marquardt algorithm in the three stages, training, testing and validation. We note that the neural network has given a good result (smallest value of mse) after 24 iterations. 


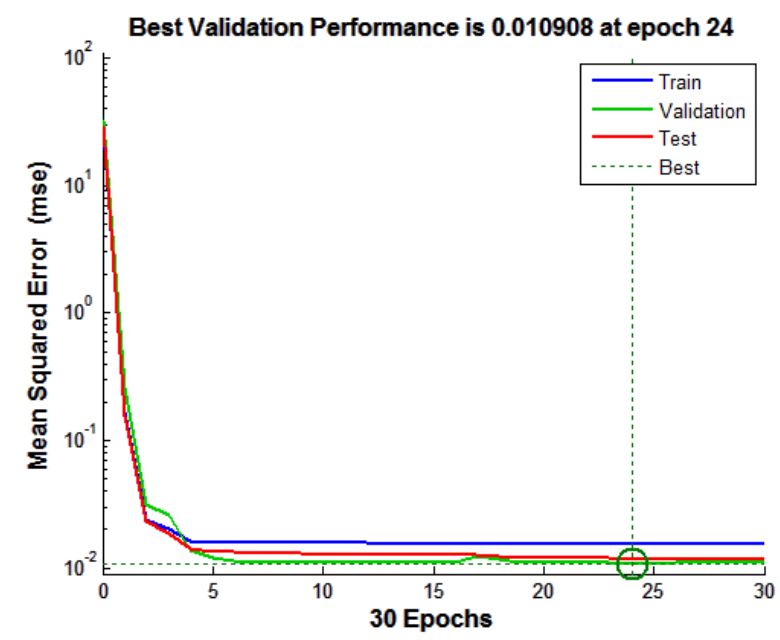

Fig. 9. The variations of the mean squared error (mse) in the network learning phase

To highlight the relevance of the used neural network and algorithm; We reported on figures 10,11 and 12 the obtained depths values by the network (ordinate $y$-output) as function of the desired depths (abscissa $x$-target) for the three data sets, training, validation and testing. The linearity between the $x$ and $y$ is highlighted by the Pearson coefficient $R(x, y)$ (Eq. 6) [15].

$$
R(X, Y)=\frac{\operatorname{cov}(X, Y)}{\sqrt{\operatorname{Var}(X) \operatorname{Var}(Y)}}
$$

A good correspondence between the ordinates and the abscissas is translated by $\mathrm{R}$ value close to one.

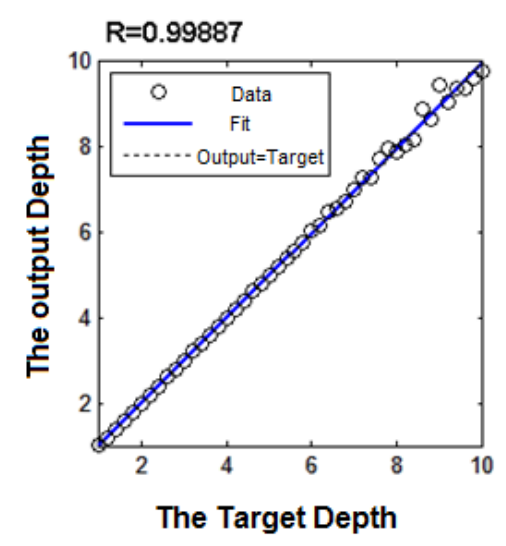

Fig. 10. The network training phase results

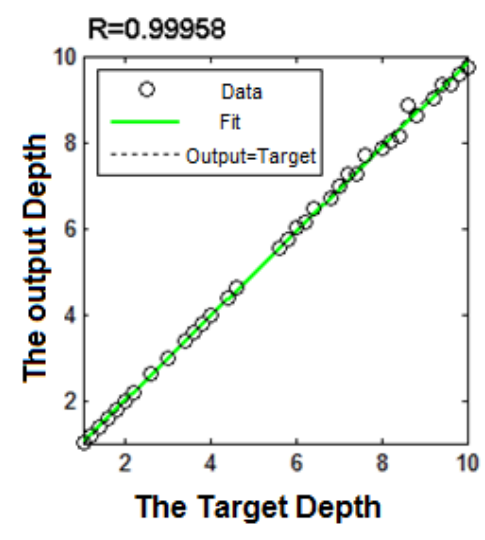

Fig. 11. The network validation phase results

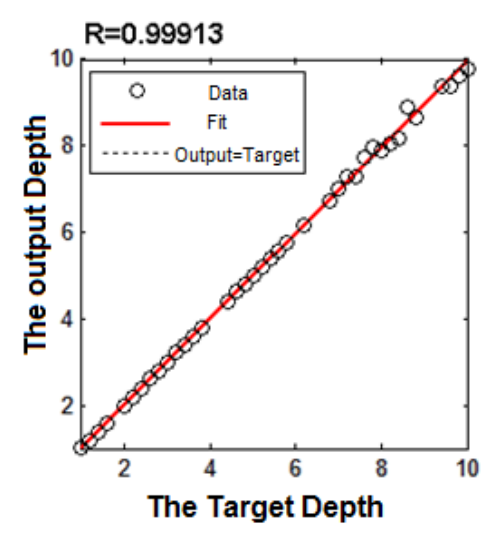

Fig. 12. The network testing phase results

The output values are represented by circles, The good correspondence (output = Target) is indicated by a dashed line and the linear regression is drawn by a dashed line. The obtained Pearson coefficients For the three sets of data, training ( $R=0.99887)$, validation $(R=0.99958)$ and test $(R=0.99913)$, shows a good linearity; We also noticed that the linear regression and the perfect match are juxtaposed and it is difficult to differentiate them; this results reflects a good match between the obtained depths (output) and the desired depths (target). These figures show that the chosen network has been successfully trained; and therefore confirm the robustness of the established neural model in defects depths prediction with the thermal contrast $\mathrm{C}\left(\mathrm{t}_{\mathrm{a}}=0.5 \mathrm{~s}\right)$ in network input. 
Table 2. Gap between estimated depths by our neural network and the desired depths

\begin{tabular}{|c|c|c|c|c|c|c|c|c|c|c|}
\hline $\begin{array}{c}\text { The desired } \\
\text { depth (mm) }\end{array}$ & 2.7 & 2.9 & 3.3 & 3.9 & 4.3 & 4.9 & 5.3 & 5.9 & 6.3 & 6.9 \\
\hline $\begin{array}{c}\text { The estimated } \\
\text { depth (mm) }\end{array}$ & 2.705 & 2.876 & 3.305 & 3.876 & 4.308 & 4.906 & 5.263 & 5.850 & 6.152 & 6.738 \\
\hline Gap (\%) & 0.17 & 0.84 & 0.15 & 0.61 & 0.17 & 0.12 & 0.68 & 0.84 & 2.34 & 2.35 \\
\hline
\end{tabular}

We reported in table 2 the new values of depths estimated by our neural network and the desired depths. These depths were not used in the learning process. We note that the provided depths by the neural network are close to the target ones. The gap is less than $3 \%$.

\section{Experimental validation}

To confirm the robustness of the established neural model in defects depths predicting using the thermal contrast $\mathrm{C}\left(\mathrm{t}_{\mathrm{a}}=0.5 \mathrm{~s}\right)$ as input. We calculated the standard thermal contrast from the measured temperatures[12] on an aluminum sample which contains defects located at $3 \mathrm{~mm}, 4 \mathrm{~mm}$ and5 $\mathrm{mm}$ (figure 13 ).

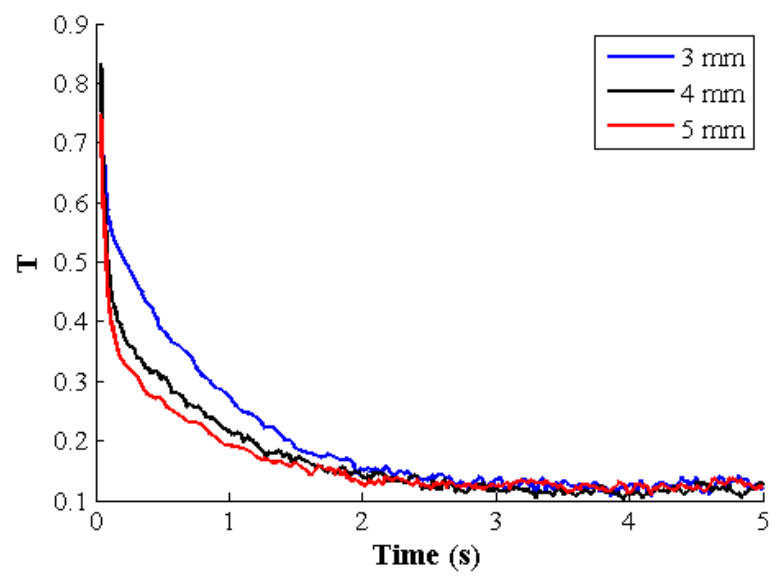

Fig. 13. Experimental Surface temperature variation of aluminum sample with defects placed at different depths.

Unlike other researchers, for each depth, we used the experimental thermal contrast $\mathrm{C}$ at the instant ta $=0.5 \mathrm{~s}$ as input in our artificial neural network. In table 4 we have transcribed the real depths values and the estimated depths values by our neural network.

Table 4. Comparisons between the estimated depths by the network and the real depths

\begin{tabular}{|c|c|c|}
\hline $\begin{array}{c}\text { The real defect } \\
\text { depth }(\mathrm{mm})\end{array}$ & $\begin{array}{c}\text { The estimated } \\
\text { depth }(\mathrm{mm})\end{array}$ & Gap (\%) \\
\hline $3 \mathrm{~mm}$ & 3.0878 & 3 \\
\hline $4 m m$ & 4.0428 & 1 \\
\hline $5 \mathrm{~mm}$ & 5.1638 & 3 \\
\hline
\end{tabular}

Table 4 confirms the effectiveness of the proposed method with experimental measurements of the surface temperature. Indeed, gaps between the real defects depths and those estimated by our neural network from the thermal contrast are very low, it does not exceed $3 \%$.

The proposed method uses a single input (contrast at the instant ta) to determine the defect depth. which represents a considerable gain in computation time and memory space, and an interesting progress in the treatment of thermograms for the defects characterization. 


\subsection{1/qirt.2016.110}

\section{Conclusion}

A standard thermal contrast values treatments technique using artificial neural networks is used in predicting defects depths. The difference between the obtained depths by the neural network and the real depth is very low; it does not exceed $3 \%$. The proposed method requires less data processing, which is a progress in data processing of the pulsed thermography.

\section{REFERENCES}

[1] Henrique Fernandes, Hai Zhang, Clemente Ibarra-Castanedo, Xavier Maldague,Fiber orientation assessment on randomly-oriented strand composites by means of infrared thermography,Composites Science and Technology 121 (2015) 25-33.

[2] A.S. Nazmul Huda, Soib Taib , Application of infrared thermography for predictive/preventive maintenance of thermal defect in electrical equipment Applied Thermal Engineering 61 (2013) 220-227.

[3] S.Dudzik, Analysis of the accuracy of a neural algorithm for defect depth estimation using PCA processing from active thermography data Infrared Physics \& Technology 56 (2013)1-7.

[4] S.Dudzik, Two-stage neural algorithm for defect detection and characterization uses an active thermography, Infrared Physics \& Technology 71 (2015) 187-197.

[5] Elhassnaoui, A. Elballouti, S. Sahnoun, Reconstruction of a triangular geometry defects in inaccessible face by a model of active thermography, J. Optoelectron. Adv. Mater. 15, 5-6, (2013) 447.

[6] A. Elhassnaoui, S. Sahnoun, A three-dimensional reconstruction algorithm for pulsed thermography, J. Mater. Environ. Sci. 5 (4) (2014) 983-988.

[7] S. Lungin et U. Netzelmann, NDT E Int. 38, 485 (2005).

[8] Vladimir P. Vavilov, Douglas D. Burleigh, Review of pulsed thermal NDT: Physical principles, theory and data processing NDT\&E International 73 (2015) 28-52.

[9] Ben Krose,Patrick van der SmagtAn introduction to neural networks,8th ed., University of Amsterdam, Faculty of Mathematics \& Computer Science,, November 1996.

[10] D.Rumelhart, G.E.Hinton, et R. J. Williams, 1986.Learning Internal Representations by Error Propagation, Volume 1, 318-362.Cambridge,MA,USA :MIT Press.

[11] Stacy L. Ozesmi , Can O. Tanb, Uygar Ozesmi , Methodological issues in building, training, and testing artificial neural networks in ecological applications,ecological modelling 195 (2006) 83-93.

[12] Z. Zeng, C. Li, N. Tao, L. Feng, C. Zhang, Absolute peak slope time based thickness measurement using pulsed thermography, Infrared Physics \& Technology 55 (2012) 200-204.

[13] X.P.V. Maldague, Theory and Practice of Infrared Thermography for Nondestructive Testing, Willey Interscience (2001) 198-199.

[14] Cybenko, G. ,Approximation by superposition of a sigmoidal function, Mathematics of Control, Signals, and System, 2,(1989), pp. 303-314.

[15] Milton, J.S., Tsokos, J.O., Statistical Methods in the Biological and Health Sciences. McGraw-Hill, Tokyo, (1983), pp. $333-380$. 